\title{
STUDY ON CLINICAL PROFILE OF PATIENTS WITH RETINAL VEIN OCCLUSION
}

\author{
Rekha Pookkatt Sankaranarayanan'1, Pappa Padmavathi², Shalos Nova³, Sudha Vaikkakara ${ }^{4}$ \\ ${ }^{1}$ Assistant Professor, Department of Ophthalmology, Government Medical College, Thrissur. \\ ${ }^{2}$ Assistant Professor, Department of Ophthalmology, Government Medical College, Thrissur. \\ ${ }^{3}$ Assistant Surgeon, DHS. \\ ${ }^{4}$ Associate Professor, Department of Ophthalmology, Government Medical College, Thrissur.
}

ABSTRACT

\section{BACKGROUND}

The aim of this study is to evaluate the clinical profile of patients with retinal vein occlusion and relate to hypertension diabetes, hyperlipidaemia and open angle glaucoma.

\section{MATERIALS AND METHODS}

The study was conducted on patients attending the Ophthalmology OPD of Thrissur Medical College during a period of one year. Screening for risk factors like hypertension, diabetes and open angle glaucoma was done. All patients were tested for BCVA, Slit lamp, IOP, Gonioscopy and Fundus. Laboratory examination for haematological parameters was done in all cases and frequent followup done.

\section{RESULTS}

Retinal vein occlusions are a disease of the elderly population. Non-ischaemic CRVO is more common than ischaemic type. Most important risk factors identified were hypertension, diabetes and hyperlipidaemia.

\section{CONCLUSION}

Initial visual acuity is a good predictor of final visual outcome.

\section{KEYWORDS}

Retinal Vein Occlusion, Visual Outcome, Sequelae.

HOW TO CITE THIS ARTICLE: Sankaranarayanan RP, Padmavathi P, Nova S, et al. Study on clinical profile of patients with retinal vein occlusion. J. Evolution Med. Dent. Sci. 2017;6(51):3885-3889, DOI: 10.14260/Jemds/2017/840

\section{BACKGROUND}

Retinal vein occlusion (RVO) defined as a retinal vascular disorder characterised by engorgement and dilatation of the retinal veins with secondary, mostly intraretinal haemorrhages and intraretinal oedema, retinal ischaemia including cotton wool spots, retinal exudates and macular oedema. ${ }^{1-8}$ As soon as the foveal region is affected by macular oedema, central visual acuity (VA) drops, leading to a progressive, or acute, painless loss in vision.

RVO is the second most common cause of reduced vision due to retinal vascular disease, after diabetic retinopathy, 9,10 yet there is marked controversy on their pathogeneses, clinical features and particularly their management. This is because the subject is plagued by multiple misconceptions. The exact pathogenesis of RVO remains unclear.

There are differences in role of each risk factor in pathogenesis of CRVO and BRVO. Owing to its multifactorial nature, management of this condition remains a challenge. Occurrence of central retinal vein occlusion is followed by another occlusion occurring in either eye and thereby identifying the risk factors, the risk of another occlusion may be dramatically reduced.

Financial or Other, Competing Interest: None.

Submission 04-02-2017, Peer Review 21-03-2017,

Acceptance 27-03-2017, Published 24-06-2017.

Corresponding Author:

Dr. Pappa Padmavathi,

Vaishnavam, White field,

Puthurkkara, Ayyanthole P. O, Thrissur-3.

E-mail: pappavinod@gmail.com

DOI: $10.14260 /$ jemds $/ 2017 / 840$
Earlier studies have shown the correlation between RVO and the risk factors and more studies are necessary to know risk factors better in this disease to determine a specific protocol for the treatment and prophylaxis of RVO.

Following up patients with RVO helps to better understand natural history of RVO, causes of defective vision, response to treatment and reasons for outcome. There is a need for further research to better understand the epidemiology and design appropriate prevention and treatment of RVO.

\section{Aims and Objectives}

1. To study the clinical profile of patients with retinal vein occlusion (RVO).

2. To determine the relationship of RVO to Systemic Hypertension, Diabetes Mellitus, Hyperlipidaemia, Obesity, Smoking, Polycythaemia, and Glaucoma.

\section{MATERIALS AND METHODS}

The study was conducted on patients who attended Ophthalmology OPD, Govt. Medical College, Thrissur diagnosed as retinal venous occlusion during the period of study.

\section{Study Design \\ Descriptive study}

Period of Study

January 2013 to June 2014.

\section{Sample Size}

65 patients with clinical evidence of retinal vein occlusion. 


\section{Inclusion Criteria}

All diagnosed cases of retinal vein occlusion.

1. A CRVO is defined as an eye that has retinal vein haemorrhage or evidence of retinal vein occlusion and dilated venous system in all 4 quadrants.

In this study, cases of hemiretinal vein occlusion included in CRVO group.

2. A BRVO is defined as an eye that has retinal haemorrhage or other evidence of retinal vein occlusion and a dilated venous system in 1 quadrant or less of retina.

\section{Exclusion Criteria}

Participants with any of the following conditions are ineligible.

A. Recent major systemic illness, evidence of vasculitis, renal, hepatic, thyroid disease, cardiomyopathy, pregnancy, psychiatric illness, anticonvulsant therapy.

B. Patients with media opacity whose evaluation of fundus is not possible like mature cataract and patients with glaucomatous optic atrophy will be excluded.

Recurrence of RVO defined as occurrence of haemorrhage and retinal oedema along another quadrant in the same or fellow eye in case of BRVO and along the central retinal vein of fellow eye in case of CRVO.

A detailed history with regard to onset and duration of visual loss, past history with relevance to systemic diseases like diabetes, hypertension, hyperlipidaemia and ocular risk factors like POAG were noted.

Ocular examination including recording of best corrected visual acuity using Snellen's chart, measurement of intraocular pressure by applanation tonometry, slit lamp examination for rubeosis iridis, gonioscopy for new vessels of angle were done. Detailed fundus examination by slit lamp biomicroscopy and indirect ophthalmoscope done for evidence of macular oedema, cotton wool spots and searched thoroughly for neovascularisation of posterior segment.

As differentiation between ischaemic and non-ischaemic is difficult, features typical of ischaemic CRVO like relative afferent papillary defect was used as a tool. SD-OCT was done in all patients who presented with initial poor acuity and repeated at frequent intervals to know extent of macular oedema and structural damage. Fundus fluorescein angiography was done in selected patients to rule out extent of retinal capillary non-perfusion and macular ischaemia who fail to improve vision.

Laboratory examination including complete haemogram, fasting blood sugar and serum lipid profile were done as basic investigations. In older patients, carotid Doppler and ECG were taken and in patients younger than 50 years of age, ANA, serum homocysteine, and peripheral smear were done. Patients were followed up monthly for at least 6 months. During each visit BCVA, slit lamp examination, IOP measurement, gonioscopy and fundus examination were recorded and sequelae like macular oedema, fundus neovascularisation, NVI and NVA, neovascular glaucoma were noted. SD OCT repeated quite often to assess response to treatment especially in patients with macular oedema.

\section{RESULTS}

A total of 65 patients with retinal vein occlusion were included in study - 130 eyes of patients; 33 male and 32 female; 29 cases of CRVO and 36 cases of BRVO.

Age and Sex distribution of Central Retinal Vein Occlusion

Age of patients who presented with retinal vein occlusion varied from 30 to 80. Maximum incidence of CRVO was in 50 - 59 years age groups. A significant proportion (67\%) of CRVO presented at younger age of $<60$ years. Females were predominantly (62\%) affected at younger age.

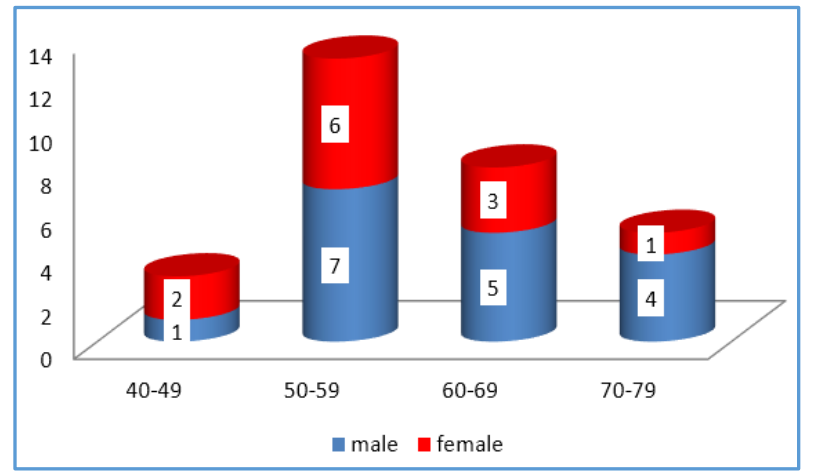

Age Distribution of CRVO

\section{Laterality}

The left eye was involved more commonly (55\%).

Among the patients with CRVO, 78\% presented with sudden painless visual loss. In $10 \%$ of patients CRVO was incidentally detected. Most of patients who presented with sudden painless loss of vision had RAPD suggestive of ischaemic CRVO.

\section{Associated Factors}

CRVO is said to be associated with various systemic disorders. In our study, hypertension was the commonest. It was seen in $34 \%$ of patients. $23 \%$ patients were diabetic. Some of hypertensive cases in the study were newly detected and started on regular treatment. One most important investigation was serum lipid profile. $34 \%$ had elevated serum cholesterol level. $10 \%$ patients had elevated homocysteine. Local risk factors were also noted, includes POAG in 2 cases of CRVO (10\%) and combined mechanism glaucoma and NTG in one each (3\%).

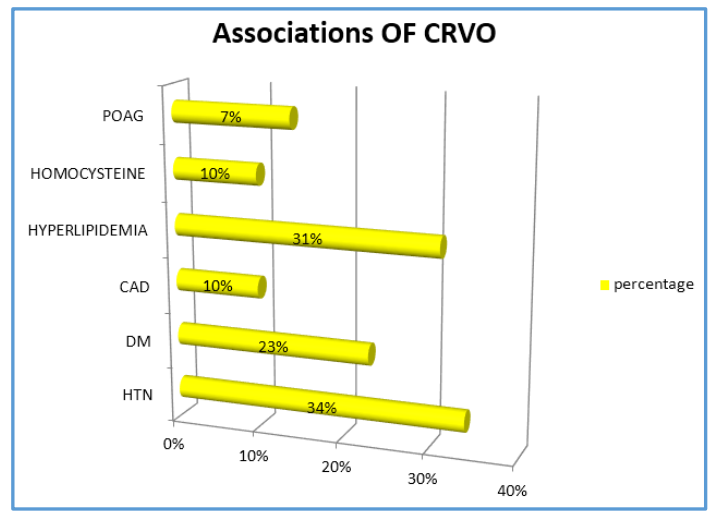

Figure 1. Graph Depicting Association of CRVO

\section{Visual Outcome of CRVO}

The visual acuity was closely monitored. $59 \%$ of Patients with CRVO presented with poor initial visual acuity of $<6 / 60$ of 
which $41 \%$ not improved even at 6 months of followup. Macular oedema was the leading cause of defective vision. Visual acuity worsened with onset of neovascular glaucoma and all these patients started on treatment according to standard treatment protocol and anti-glaucoma medications and IOP were made under control.

\begin{tabular}{|c|c|c|c|}
\hline Visual Acuity & Initial & At 3 Months & At 6 Months \\
\hline CF-5/60 & $17(59 \%)$ & $15(52 \%)$ & $12(41 \%)$ \\
\hline $6 / 60-6 / 18$ & $10(34 \%)$ & $11(38 \%)$ & $12(41 \%)$ \\
\hline$>6 / 18$ & $2(7 \%)$ & $3(10 \%)$ & $5(18 \%)$ \\
\hline \multicolumn{4}{|c|}{ Table 1. Visual Outcome in CRVO } \\
\hline
\end{tabular}

Rubeosis iridis was noted in 3 patients (25\%) with ischaemic CRVO within four to six months and they went on to develop neovascular glaucoma one to two months later. Patients were treated with standard treatment protocol of RVO and kept under close followup. Consecutive Optic atrophy and collaterals on disc was noted in one (8\%) patient. Two (16\%) patients developed NVE and closely monitored for NVI and NVA. Vision remained poor in 8 patients (67\%), out of which 4 cases $(50 \%)$ were due to persistent macular oedema.

In patients with CRVO who regained good vision at six months, sequelae noted were formation of microaneurysm IRMA and collaterals at non-perfused areas. One patient developed NVE and pre-retinal haemorrhage. Two patients developed rubeosis iridis and NVG. Three cases $(18 \%)$ of non-ischaemic CRVO changed to ischaemic CRVO during followup.

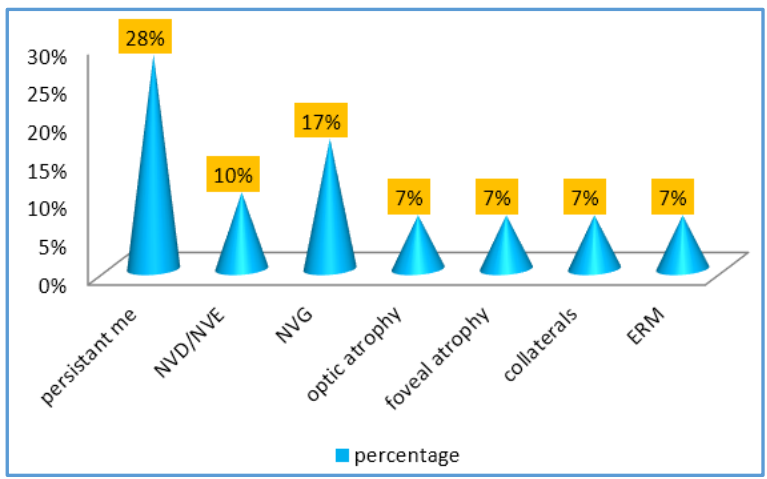

Figure 2. Sequelae of CRVO

\section{BRVO}

\section{Distribution}

Among BRVO group, a total of 36 patients included in the study, 17 male and 19 female. Majority of BRVO affected superotemporal branch of retinal vein (64\%). 7 cases were recurrent cases of BRVO affecting major branches of retinal vein. One patient presented with features of neovascular glaucoma.

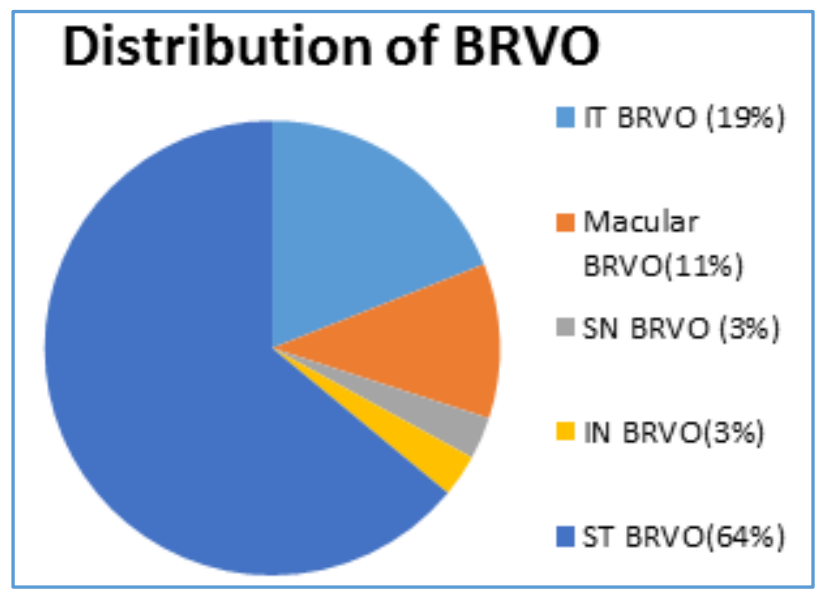

Figure 3. Distribution of BRVO

\section{Laterality}

Left eye was more commonly affected (53\%).

\section{Age and Sex Predilection}

Similar to Patients with CRVO, BRVO also found more between 51-59 of age with females more affected at younger age. There was no sex predilection in major BRVO with equal incidence among both groups. Of the 4 cases of macular BRVO $75 \%$ affected were female.

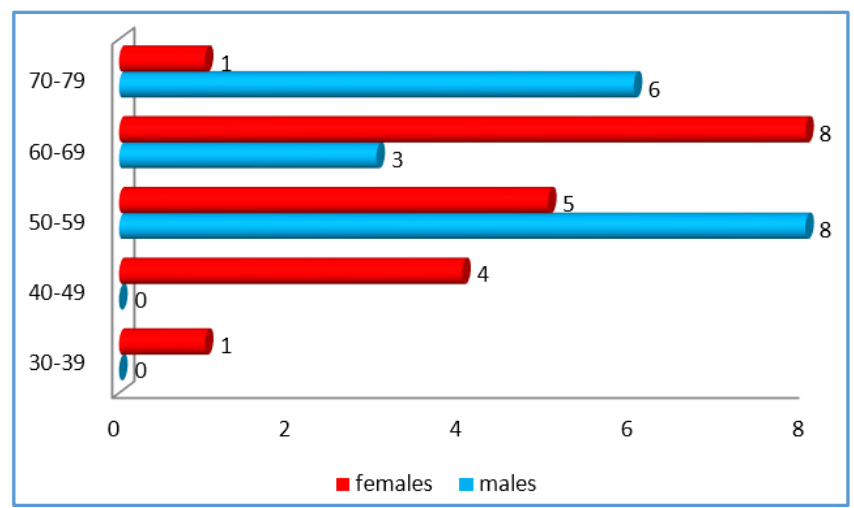

Figure 4. Age and Sex Distribution of BRVO

Among associations of BRVO, uncontrolled hypertension was found to be major one (58\%). Diabetes was found to be affecting 30\% patients. POAG seen in 4 patients. Cholesterol level rose in 11 (30\%) patients and 5 (14\%) patients had associated atherosclerotic vascular disorders. Two patients had raised homocysteine levels.

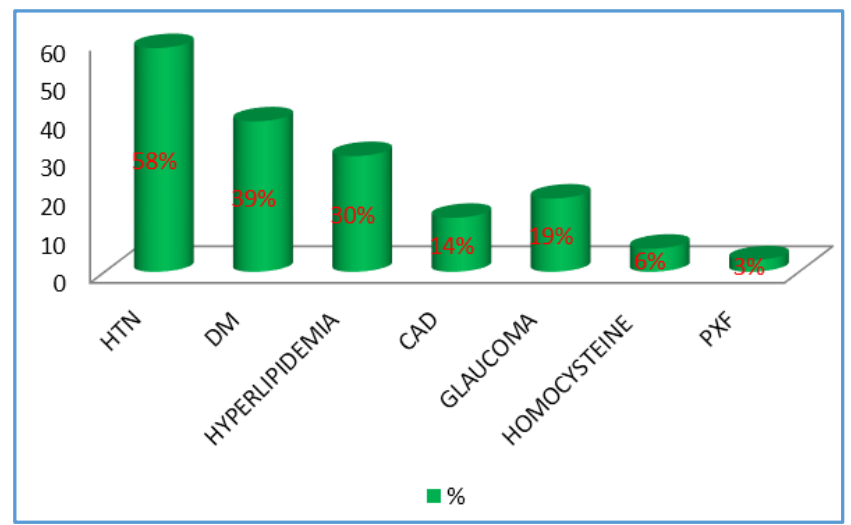

Figure 5. Associations of BRVO 
Visual acuity at presentation varied from $1 \mathrm{mcf}$ to $6 / 9$. Major causes of defective vision was macular oedema of which $30 \%$ of patients responded well to standard treatment protocol. 3 patients (8\%) showed further diminution of vision within 6 months. Four persons (11\%) with initial visual acuity $<6 / 60$ did not improve even during the study period, found to be due to macular ischaemia and persistent macular oedema. Patients with initial visual acuity $>6 / 12$ found to have good vision throughout.

\begin{tabular}{|c|c|c|c|}
\hline Visual Acuity & Initial & At 3 Months & At 6 Months \\
\hline$<6 / 60$ & $7(19 \%)$ & 5 & $4((11 \%)$ \\
\hline $6 / 60-6 / 18$ & $14(39 \%)$ & 15 & $12(33 \%)$ \\
\hline $6 / 18-6 / 12$ & $12(33 \%)$ & 13 & $15(42 \%)$ \\
\hline$>6 / 12$ & $3(8 \%)$ & 3 & $5(14 \%)$ \\
\hline \multicolumn{3}{|c|}{ Table 2. Visual Outcome of BRVO over 6 Months }
\end{tabular}

Sequelae to BRVO includes recurrence of RVO - 5\% patients developed recurrent BRVO and 3\% of patients developed HRVO in same eye and one patient develops STBRVO in other eye on followup. 3 patients (8\%) developed NVD and NVE with FFA showed areas of capillary nonperfusions. One person developed NVI and endovascular glaucoma.

$11 \%$ of patients were found to have persistently low vision of which $5 \%$ patients developed persistent macular oedema not responding to standard treatment protocol and $5 \%$ patients had foveal atrophy. Collaterals along the occluded vessels developed in 4 patients $(11 \%)$ showed improvement of vision and one patient developed consecutive optic atrophy.

\section{DISCUSSION}

\section{CRVO Group}

Even though CRVO has been reported in all age groups it is predominantly a disease of the elderly with more than $90 \%$ of CRVO patients being older than 50 years. In our study, the mean age of presentation of CRVO was 60.4 years. In one large case series study by Hayreh et al, the mean age of onset of vein occlusion was 63 years. ${ }^{1}$ He also noted that nonischaemic CRVO is seen more common in younger age compared to ischaemic CRVO.

In most of the published studies of CRVO, males are more affected than females. ${ }^{2}$ In our series also males are more affected than females. But females are commonly affected at younger age.

Commonest symptom is sudden painless visual loss. Compared to right, left eye was more affected. According to a study by Hayreh et al in 544 eyes with non-ischaemic CRVO and 191 eyes with ischaemic, ischaemic CRVO showed a trend toward more left eye involvement, with no difference in the non-ischaemic CRVO. 3.70\% of patients with CRVO presented with initial poor visual acuity of $<6 / 60$, persistent to have low vision even at 6 months of followup might be ischaemic type of CRVO. According to Hayreh, vision in ischaemic CRVO was $<6 / 60$ in majority of cases.

$31 \%$ of the patients in CRVO group had persistently low vision. $40 \%$ of patients with good initial visual acuity had maintained their vision. $10 \%$ of patients had drop in vision. So it is observed that initial visual acuity is an important factor determining the final visual outcome with significant odds ratio of 9.644. CVOS study has reported that visual acuity outcome in CRVO was largely dependent on initial acuity. ${ }^{11}$ Eyes with good initial vision have a good chance of maintaining excellent vision while a poor visual outcome is seen in those who had poor initial acuity.

$17 \%$ of patients with CRVO developed NVI within 4 months and they all developed NVG within 2 months of appearance of new vessels, most probably ischaemic type of CRVO. According to literature, incidence of rubeosis is $20 \%$ among CRV0.11,12 Among ischaemic eyes, rubeosis with or without NVG occur in 45 to $80 \%$ and this usually develops by 4-7 months of the occlusion. Neovascularisation of optic disc or retina is a rare complication but has been reported in $24 \%$ of ischaemic CRVO.13

Various associations have been described for CRVO. They may be local (in the eye or central retinal vein), systemic, or haematologic. It is well established that CRVO is significantly more common in patients with raised intraocular pressure (IOP) and glaucoma. Three of our patients (10\%) with CRVO had POAG and one patient (3\%) had NTG, although it is not statically significant. Study by Dreyden et al showed $40 \%$ or more of patients with CRVO had pre-existing OAG or this condition developed during followup. ${ }^{5}$ According to Hayreh et al, IOP of more than $22 \mathrm{mmHg}$ was seen in $22 \%$ of patients with CRVO.

In our study, hypertension, DM, hyperlipidaemia and coronary artery diseases were the systemic conditions associated with CRVO. Although hypertension was the major risk factor in CRVO group but it is statistically insignificant. Significant associations of diabetes mellitus, cardiovascular disease, and arterial hypertension in ischaemic CRVO and arterial hypertension in non-ischaemic CRVO were described by the Eye Disease Case-Control Study Group.

Various types of venous occlusions are associated with haematological abnormalities. Among this Lupus anticoagulant factor, antiphospholipid antibody syndrome, protein $\mathrm{C}$ deficiency and activated protein $\mathrm{C}$ resistance have been cited as risk factors for CRVO in young. $7,8,9$ Even though in our study few cases of CRVO $<50$ years presented with elevated homocysteine and dyslipidaemia, no specific aetiology could be found in these patients as the values are not statistically significant. Despite extensive investigations CRVO in many of young patients have no identifiable underlying cause.

\section{BRVO Group}

In our study, majority of BRVO affects major branches of retinal vein especially superotemporal retina (50\%) and 4 cases were macular BRVO. According to Lang et al, in $66 \%$ of eyes with BRVO, there is occlusion of the major branch in the superotemporal quadrant followed by $22-43 \%$ of eyes with occlusion of the major branch in the inferotemporal quadrant. ${ }^{9}$ Owing to absent subjective BRVO symptoms in nasal quadrants, the diagnosis of occlusion in this localisation is mostly accidental and therefore rare. ${ }^{1}$

7 cases were recurrent cases (19\%) of BRVO. One case presented with features of neovascular glaucoma. According to Hayreh, the cumulative probability of developing a second episode of occlusion in the other eye within 4 years is about $7 \%$ in patients with BRVO. ${ }^{4}$

BRVO also found more between 51-60 years with females affected more (53\%) at younger age group may be due to sedentary lifestyle and decrease in protective effect of hormones. Hayreh et $\mathrm{al}^{4}$ investigated the demographic 
characteristics of various types of RVO in 1108 patients (1229 eyes). In this study, a male: female ratio of 1.2:1 was noted in a group of patients with RVO.

VA is a very sensitive indicator of the oxygen situation in the macula. For this reason, pre-treatment VA may be an important prognostic factor. In our study, visual acuity at initial presentation varied from $1 \mathrm{mcf}$ to $6 / 9.54 \%$ of patients presented with initial poor BCVA not improved vision with standard treatment protocol found to be mainly due to macular ischaemia and persistent macular oedema. One study investigated the visual prognosis in 246 eyes with BRVO divided into two groups: with and without laser treatment. The obtained analysis illustrates that in the group of eyes with an initial VA 20/50 or better, no eye (not receiving laser treatment) and only $13 \%$ eyes (had undergone laser treatment) had a final VA of $20 / 200$ or worse, whereas in the group of eyes with an initial VA $20 / 200$ or worse, $83 \%$ of eyes (not receiving laser treatment) and $50 \%$ of eyes (had undergone laser treatment) had this unsatisfactory final VA.

Sequelae to BRVO includes recurrence of RVO - 5\% patients developed recurrent BRVO and 3\% of patients developed HRVO in same eye and one patient developed STBRVO in another eye on followup.

$3 \%$ of patients developed NVD and 5\% patients NVE with areas of capillary non-perfusions in FFA responded well to PRP. One person (2\%) developed NVI and NVG. According to literature, NVD develops in approximately $10 \%$ of eyes, and NVE occurs in approximately $20 \%$ of eyes. NVI and NVG are uncommon and occur in only approximately $1 \%$ of affected eyes.

Among systemic associations of BRVO, uncontrolled hypertension was found to be a major one (58\%). Cholesterol raised in 11 patients (30\%) and 5 patients (14\%) had associated occlusive atherosclerotic vascular disorders, but the values are statically insignificant. ${ }^{14}$ Systemic hypertension, diabetes mellitus, atherosclerosis, and smoking are reported to be more common in patients with RVO.10

\section{CONCLUSION}

1. Incidence of retinal vein occlusion is mainly seen in elderly population ( $>50$ years), but now increasing in younger individuals.

2. No sex predilection found in CRVO and BRVO, but macular BRVO found more in females.

3. Initial visual acuity is found to be a significant factor determining final visual outcome especially in CRVO.

4. Major cause of defective vision was macular oedema which in most of cases responded well to standard treatment protocol, but in some cases persistent oedema led to structural damage to fovea.

5. Sequelae to RVO mainly include neovascularisation in the anterior and posterior segment of eye especially in CRVO. Others include foveal and optic atrophy and in some cases development of collaterals which helped in improvement of vision.

6. Hypertension is a major systemic risk factor seen in both CRVO \& BRVO, diabetes and hyperlipidaemia came second. Homocysteine was seen as a risk factor in younger population. POAG was seen in both RVOs as an ocular risk factor.

\section{Summary}

RVO is a multifactorial disease, common among elderly population, with significant rise of occurrence in the young and females are affected more at younger age. BRVO is more common than CRVO. Hypertension is the major association in elderly and no specific aetiology found for RVO in younger patients. Eyes with good initial vision have a better chance of maintaining excellent vision while a poor visual outcome is seen in those who had poor initial acuity. Early detection and proper control of associations have a major role in prevention and recurrence of RVO. Frequent followup is needed for patients with RVO for prevention and treatment of sequelae.

\section{Limitations}

1. A larger sample size and more duration of study might give more details of risk factors and better basement of natural history of retinal venous occlusion.

2. In young RVO cases more detailed investigations are needed to know the risk factors.

\section{REFERENCES}

[1] Hayreh SS. Classification of central retinal vein occlusion. Ophthalmology 1983;90(5):458-74.

[2] Hayreh SS, Zimmerman MB, Podhajsky P. Incidence of various types of retinal vein occlusion and their recurrence and demographic characteristics. Am J Ophthalmol 1994;117(4):429-41.

[3] Hayreh SS. Retinal vein occlusion. Indian J Ophthalmology 1994;42(3):109-32.

[4] Dryden RM. Central retinal vein occlusions and chronic simple glaucoma. Arch Ophthalmol 1965;73(5):659-63.

[5] Risk factors for central retinal vein occlusion. The Eye Disease Case-Control Study Group. Arch Ophthalmol 1996;114(5):545-54.

[6] Larsson J, Olafsdottir E, Bauer B. Activated protein C resistance in young adults with central retinal vein occlusion. Br J Ophthalmol 1996;80(3):200-2.

[7] Larsson J, Sellman A, Bauer B. Activated protein C resistance in patients with central retinal vein occlusion. Br J Ophthalmol 1997;81(10):832-4.

[8] Vine AK, Samama MM. Screening for resistance to activated protein $\mathrm{C}$ and the mutant gene for factor $\mathrm{V}:$ Q506 in patients with central retinal vein occlusion. Am J Ophthalmol 1997;124(5):673-6.

[9] Hayreh SS. Central retinal vein occlusion. In: Mausolf FA. edr. The Eye and systemic disease. $2^{\text {nd }}$ edn. St. Louis: CV Mosby 1980:223-75.

[10] The Central Vein Occlusion Study Group. A randomized clinical trial of early panretinal photocoagulation for ischemic central vein occlusion. Ophthalmology 1995;102(10):1434-44.

[11] Sinclair SH, Gragoudas ES. Prognosis for rubeosis iridis following central retinal vein occlusion. $\mathrm{Br} \mathrm{J}$ Ophthalmol 1979;63(11):735-43.

[12] Brown GC, Moffat K, Cruess A, et al. Cilioretinal artery obstruction. Retina 1983;3(3):182-7.

[13] Central Vein Occlusion Study Group. Baseline and early natural history report. Arch Ophthalmol 1993;111(8):1087-95.

[14] Quinlan PM, Elman MJ, Bhatt AK, et al. The natural course of central retinal vein occlusion. Am J Ophthalmol 1990;110(2):118-23. 